\title{
P-CARDS: Proposta de um protocolo formal para carregamento e controle de qualidade de dados sísmicos e de poços na interpretação sísmica.
}

\begin{abstract}
Paulo Augusto Vidigal D. Souza, GETA/CPGG/UFBA; Daniel Bono R. Vilas Boas, GETA/CPGG/UFBA; Michael Holz,
\end{abstract} GETA/CPGG/UFBA

Copyright 2016, SBGf - Sociedade Brasileira de Geofísica

Este texto foi preparado para a apresentação no VII Simpósio Brasileiro de Geofísica, Ouro Preto, 25 a 27 de outubro de 2016. Seu conteúdo foi revisado pelo Comitê Técnico do VII SimBGf, mas não necessariamente representa a opinião da SBGf ou de seus associados. É proibida a reprodução total ou parcial deste material para propósitos comerciais sem prévia autorização da SBGt.

\section{Abstract}

The import of seismic data is the first and fundamental step towards seismic interpretation. Due to errors in this initial stage, difficulties and incongruities commonly arise during the interpretation phase, with errors ranging from incorrect loading of geographic coordinates to mistie problems and inadequate correlations between seismic lines and wells. The seismic interpreter, especially at junior level, transits intuitively and with great difficulty trough the data loading phase, due to the absence of a publication that systematizes the procedures and guides the geophysicist through this crucial step prior to interpretation. Formal publications are inexistent in the literature or are restricted to internal company handbooks. Therefore, the aim of this paper is to present a formal proposal of the so-called P-CARDS, "protocol for loading and quality control of seismic data".

\section{Introdução}

Durante a interpretação sísmica, é comum surgirem dificuldades e com elas o questionamento da confiabilidade do dado. A causa pode estar associada, além de razões geológicas, com um carregamento de dados realizado de maneira incorreta. Um controle de qualidade deve também auxiliar a corrigir ou melhorar dados que estejam comprometidos.

A motivação deste trabalho é que existe uma dificuldade em encontrar publicações referentes a como se deve proceder para realizar o carregamento de dados sísmicos e de poços. O novo profissional da área encontra dificuldades em diversas etapas do carregamento, perdendo assim tempo e comprometendo a interpretação que vier a fazer. Este trabalho, propõe um protocolo de carregamento de forma a agilizar e embasar o carregamento sísmico e de poços realizado (P-CARDS).

A padronização do formato dos dados sísmicos da SEG (Society of Exploration Geophysics) foi originalmente desenvolvida em 1973 e foi publicado por Barry et al. (1975). Entretanto, houveram avanços diversos na aquisição e processamento de dados geofísicos, demandando assim, constantes atualizações. O Trace Header (local de armazenamento dos parâmetros de aquisição) continha informações organizadas do byte 0 até o 180. A partir do byte 181 até o 240 era classificado como "não assinalado - para informações opcionais".

Em 2002 Norris et al. fizeram a última atualização. Nesta padronização constam informações do byte 181 até o 232 , tais como coordenadas geográficas em UTM X (181-184) e Y (185-188) dos CDPs, número das in-line (189-192) e das crosslines (193-196) em dados 3-D post-stack entre outras informações. Sobrou para as informações adicionais o intervalo dos bytes 233 ao 240. Nem sempre os dados a serem trabalhados vão obedecer essa organização, principalmente se forem dados mais antigos. Pode ocorrer também de o padrão (default) do software não ser o convencionado pela SEG.

Até a década de oitenta, as informações sobre como eram perfilados os poços e o funcionamento de suas ferramentas eram bastante confinados e pouco conhecidos. Isso começou a mudar na década de noventa e desde lá a importância de um controle de qualidade dos poços vem aumentando cada vez mais (Theys, P., 1999). Essa garantia de qualidade do dado é dividida entre empresa e contratante, onde a empresa deve garantir a qualidade e 0 contratante deve testá-la. Alguns procedimentos podem ser realizados para avaliar a confiabilidade do dado ainda que não tenha informações técnicas como mau funcionamento do equipamento, rotatividade da ferramenta, desvio do poço, etc.

Para a realização do carregamento de poços é geralmente necessário o arquivo LAS. Nem todos os poços contam com o arquivo LAS que contém, em formato de texto, as informações das curvas que foram perfiladas. O P-CARDS mostra como converter arquivos para o formato necessário para o carregamento do dado e como proceder caso conte apenas com um arquivo de imagem do perfil composto.

\section{Dados sísmicos}

Os softwares utilizados para análise das informações contidas no header dos dados sísmicos em formato SEGY são o Seisee (gratuito) e o Data Explorer da IHS.

Para o controle de qualidade dos dados sísmicos deve-se checar e relatar o tempo de gravação e a taxa de amostragem. Lacunas de informações de CDPs podem significar dado deteriorado que se perdeu durante a recuperação, portanto devem ser investigados.

Verificar se o header binário e o EBCDIC (Extended Binary Coded Decimal Interchance Code) estão completos. O dump do header (onde é possível ver a informação do traço alocada em determinado byte) deve ser examinado para ver se está em conformidade com as definições e especificações da SEG (Figura 1). 


\begin{tabular}{|c|c|c|c|}
\hline Bin $\mathrm{H}_{\mathrm{C}}$ & leader & Irace Header & Trace Data \\
\hline Trc\# 1 & & & \\
\hline ltems & Dump & & \\
\hline Format & Integer 4 & $\nabla$ & \\
\hline 1 st byte & 1 & $=$ & \\
\hline $73 \cdot 76$ & 538405 & & \\
\hline $77 \cdot 80$ & 8581700 & & \\
\hline $81 \cdot 84$ & 0 & & \\
\hline $85 \cdot 88$ & 0 & & \\
\hline $89-92$ & 65536 & & \\
\hline $93-96$ & 0 & & \\
\hline $97-100$ & 0 & & \\
\hline $101-104$ & 37 & & \\
\hline 105-108 & 0 & & \\
\hline $109-112$ & 0 & & \\
\hline $113-116$ & 1550 & & \\
\hline $117-120$ & 262144000 & & \\
\hline $121-124$ & 0 & & \\
\hline $125-128$ & 0 & & \\
\hline $129-132$ & 0 & & \\
\hline $133-136$ & 0 & & \\
\hline $137-140$ & 0 & & \\
\hline $141-144$ & 0 & & \\
\hline $145-148$ & 0 & & \\
\hline $149-152$ & 0 & & \\
\hline $153-156$ & 0 & & \\
\hline $157-160$ & 130220259 & & \\
\hline $161-164$ & 56 & & \\
\hline $165-168$ & 327682 & & \\
\hline $169-172$ & 0 & & \\
\hline $173-176$ & 0 & & \\
\hline $177-180$ & 0 & & \\
\hline $181-184$ & 538422 & & \\
\hline 185-188 & 8581818 & & \\
\hline
\end{tabular}

Figura 1: Dump do header, mostrando as informações do traço. No caso da figura escolhida, notar que as caixas marcadas na esquerda são as das coordenadas da fonte $X$ (73-76) e $Y$ (77-80) e as coordenadas $X$ e $Y$ dos CDPs, condizendo com o padrão da SEG.

As informações obtidas no controle dos dados devem ser inseridas numa tabela para que possam ser consultadas durante a interpretação. Algumas informações podem ser inseridas como sufixo, em especial 0 intervalo de amostragem (IA), como exemplo: "LEVANTAMENTO_0123.456.MIG_FIN.78_IA2" onde foi inserido a sigla "IA2" significando um intervalo de amostragem de $2 \mathrm{~ms}$ ). Essa informação pode auxiliar, por exemplo, no momento de inserir os parâmetros para um carregamento adequado sem suprimir amostras e na escolha dos parâmetros adequados para a obtenção de uma wavelet extraída do dado na geração de um sismograma sintético.

A presença de ruídos aleatórios ou não coerentes e estruturas anômalas nas seções sísmicas, que não são facilmente explicadas por processos de aquisição ou processamento sísmico, devem ser observados no controle dos dados. Posteriormente estas informações juntamente com outras que podem ser mais subjetivas como a presença de múltiplas e sorrisos de migração devem corroborar para uma melhor avaliação da confiabilidade das seções sísmicas e consequentemente da interpretação. Dessa forma pode-se sugerir um reprocessamento mais bem direcionado ao problema de maneira a saná-lo com mais objetividade e celeridade. A Tabela 1 mostra que após um controle de qualidade é possível unir diversas informações sobre cada linha sísmica de interesse, permitindo assim, tomar as devidas providencias para obter um melhor produto final.

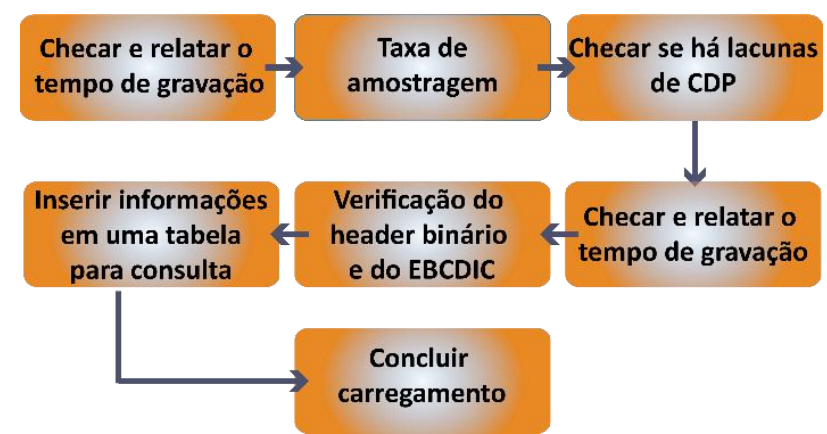

Figura 2: P-CARDS referente ao controle de qualidade a ser feito antes do carregamento de dados sísmicos.

\section{Dados de poços}

Para as conversões dos formatos na obtenção do arquivo LAS dos poços foi utilizado o software de conversão Schumberger Log Data ToolBox (gratuito). O software utilizado para realizar o carregamento dos dados sísmicos e de poços é o IHS Kingdom.

Um controle de qualidade previamente ao carregamento dos dados de poços é importante no aspecto de validação das informações recebidas, bem como na edição dos perfis geofísicos de poços. Os dados de poços, muitas vezes, contêm tanto os perfis geofísicos quanto as informações da perfuração (dados de direção e calibragem, etc.). Na etapa da interpretação sísmica, apenas são utilizados, comumente, os perfis geofísicos e o arquivo de direcionamento de poço (no caso de poços com desvios). A confecção de uma tabela com informações básicas sobre os poços é recomendada (Tabela 2).

Para o controle de qualidade das curvas dos poços é as vezes necessário conversões de formato de arquivo para o carregamento. Comumente os softwares aceitam apenas 0 formato LAS. O Arquivo LAS pode ser visualizado sem muitos recursos de software e é um arquivo ASCII, sendo menos robusto que os formatos LIS ou DLIS. Quando o arquivo chega nos formatos LIS ou DLIS, para carregar em diversos softwares de interpretação é preciso convertê-los em LAS.

Os arquivos LIS costumam vir reunidos em um único arquivo em formato TIFF para cada poço disponibilizado. Este deve ser primeiro fragmentado em uma série de outros arquivos contendo as curvas para cada poço. 
O módulo de conversão LIS para DLIS pode agora ser utilizado, com segurança, para cada arquivo. Posteriormente, os arquivos em DLIS devem ser convertidos para LAS (utilizando o módulo DLIS to ASCII), observando as unidades métricas nas conversões. Desta forma o arquivo deve estar pronto para o carregamento (Figura 3).

Após o carregamento, antes de iniciar a interpretação ainda deve ser feito uma edição dos perfis que devem auxiliar na confecção de sismogramas sintéticos (Figura 4) e permitindo assim, a amarração com a sísmica. É comum os perfis virem com valores anômalos que devem ser subtraídos do dado. É aconselhável ter um registro em relação à qualidade do log, considerando que "armadilhas" (pitfalls) na interpretação são comuns de acontecerem associadas a zonas lavadas, desmoronamentos e efeitos de invasão de fluidos de perfuração.

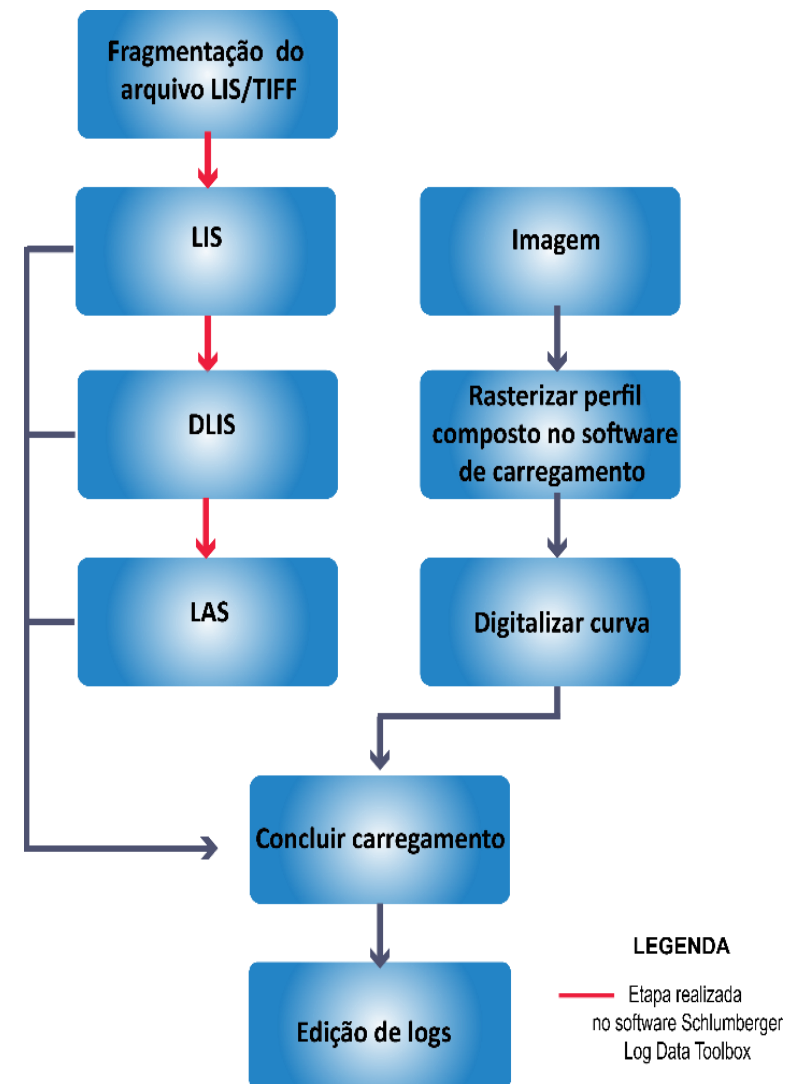

Figura 3: P-CARDS, protocolo para carregamento e edição de poços.

Os intérpretes utilizam principalmente os perfis: sônico, densidade, resistividade, raio gama e potencial espontâneo. Sendo os dois primeiros perfis essências para uma boa amarração poço-sísmica, e os demais importantes em diversas caracterizações/correlações geológicas. Em casos de ausência do perfil sônico ou de densidade, seus valores podem ser estimados através das fórmulas empíricas de Gardner et al. (1974), ou ainda, pela Fórmula de Faust (1953).
Um resumo do passo a passo da edição de logs é:

1. Retirar valores incoerentes. É comum a presença de valores incoerentes como densidades de 99999.999 $\mathrm{g} / \mathrm{cm}^{3}$ ou valores zerados. Então o primeiro passo é retirar esses valores para não alterarem o aspecto da curva.

2. Avaliar quais são os trechos desmoronados ou que não foram perfilados para ver os trechos com as medidas mais confiáveis.

3. Preencher os trechos não perfilados ou melhorar os trechos desmoronados. Isso pode ser feito em intervalos onde se tenha o perfil de resistividade para que, através da equação de Faust, 1953, se obtenha as velocidades. Outra opção, é obter as velocidades através do RHOB (densidade) utilizando a equação de Gardner et al., 1974.

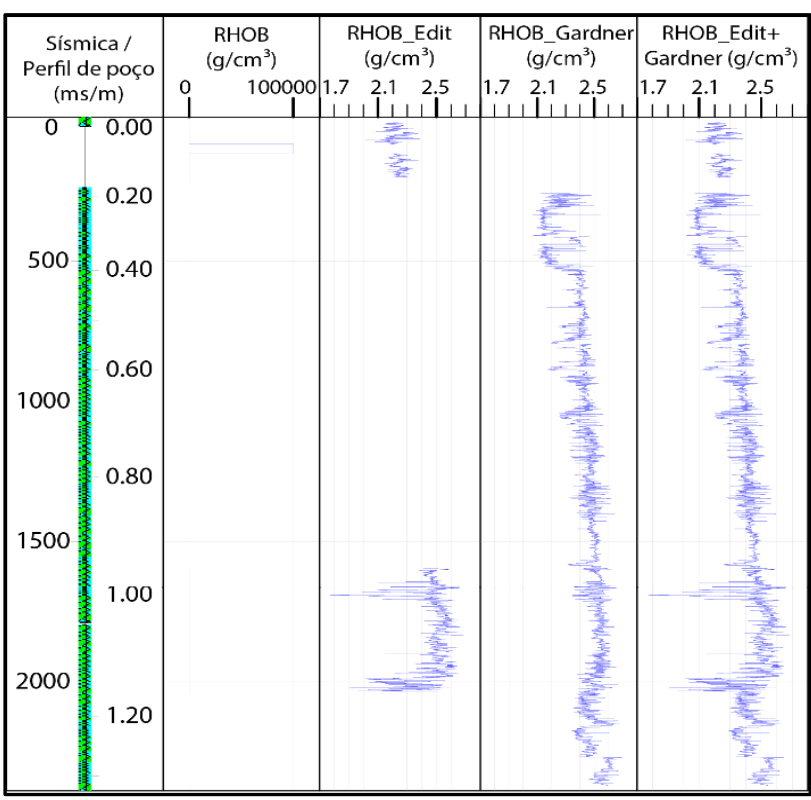

Figura 4: Edição do perfil de densidade RHOB. a) Representação da $T$-D chart. b) Perfil sem edição, com valores anômalos de densidade. c) Perfil RHOB sem valores anômalos. d) Perfil RHOB extraído do perfil sônico através da relação empírica de Gardner (1974). e) União entre os perfís medido (Editado) com o calculado onde não havia informação.

Para a amarração sísmica-poço, é aconselhável que o perfil sônico passe primeiramente por uma suavização em sua frequência de amostragem para que possa ser corrigido o efeito drifte que ocorre durante a amarração com a sísmica. O efeito drifte é a diferença de tempo de transito devido à frequência sísmica e a frequência de amostragem do perfil sônico (Cui \& Margrave, 2015). A suavização descrita aproxima mais as frequências entre os perfis de poços (que podem atingir a ordem de quilohertz) e os dados sísmicos, que, limitados pela frequência de Nyquist, usualmente interessam frequências na ordem de dezenas de hertz. 


\section{Caso específico}

Em alguns casos os dados recebidos apresentam ausência de alguns perfis geofísicos, ou ainda dos arquivos dos dados de poços digitalizados (LIS, DLIS ou LAS). Nestas situações, havendo o arquivo do perfil composto, é possível digitalizar as curvas a partir de ferramenta disponível pelo próprio software de interpretação sísmica (Figura 5). O processo se trata de capturar a imagem do perfil composto (raster log) para depois digitalizar a curva (digitize log from raster curve).

Para o carregamento de poços direcionais é preciso de informações adicionais como a inclinação, azimute, rumo e coordenada de cada profundidade em que o poço sofre algum desvio (Figura 6).

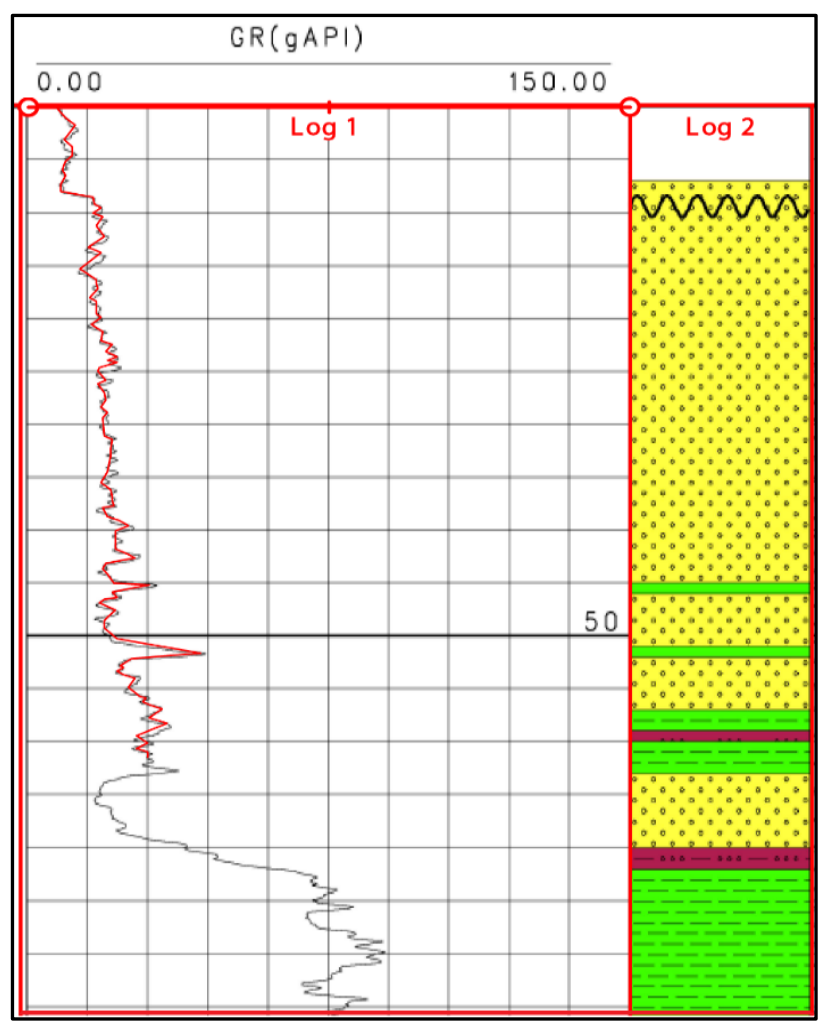

Figura 5: Trecho de um perfil composto, onde a curva de raios gama (GR) está sendo digitalizada no software Kingdom IHS, após ter sido importado em formato raster.

\section{Discussão e Conclusões}

Este trabalho traz uma descrição de como fazer um carregamento dos dados sísmico e de poços associados a um controle de qualidade e tem como conclusão que se tal etapa for realizada cautelosamente, diversos futuros problemas, como coordenadas incorretas, podem ser evitados. Através de uma tabela de controle detalhada é possível antecipar o tratamento de dados como um reprocessamento de seções sísmicas, essenciais à uma interpretação confiável. Pode ainda auxiliar a encontrar a raiz de possíveis inconsistências futuras que possam vir a surgir, permitindo assim solucioná-las mais rapidamente.

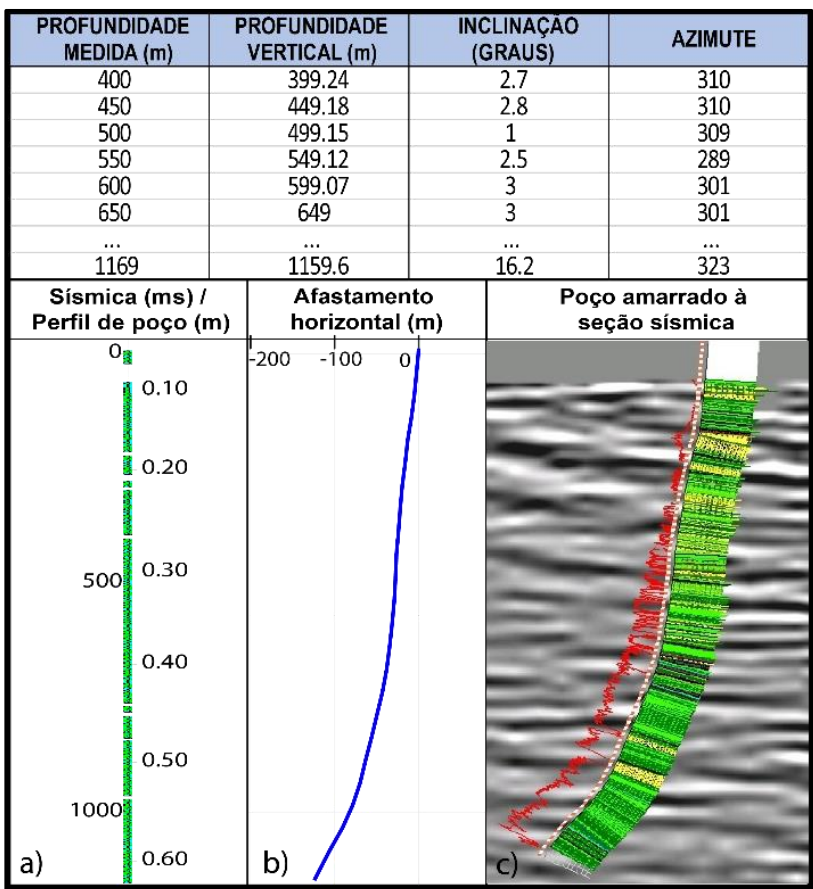

Figura 6: A tabela acima conta com as informações de inclinação e azimute para cada profundidade, medida e vertical, do poço. a) Relação tempo profundidade do poço. b) Afastamento horizontal do poço em relação à boca do poço. c) tabela Representação do poço direcional com a litologia e o perfil de raios gama amarrado à seção sísmica.

Dificuldades e imprevistos fazem parte da rotina do intérprete, que usualmente, lida com grande volume de dados e softwares que demandam conhecimento de diversos parâmetros para a sua correta utilização. A motivação inicial para a realização deste trabalho, deu-se devido a um problema de carregamento ocorrido no âmbito de trabalho ao qual esse trabalho se insere. Demandou tempo e esforço para encontrar as inconsistências. Logicamente, se o carregamento do dado sísmico for feito com as coordenadas da fonte ao invés de utilizar as coordenadas CDP, as linhas ficarão dispostas de maneira incorreta, variando o erro de acordo com o tipo de aquisição realizada. Em alguns casos a ordem pode ser da ordem de dezenas de metros, em outros pode chegar a quilômetros e principalmente no caso de linhas não retas (crooked) o erro de posicionamento é ainda maior.

Em casos em que se tem dúvidas sobre o carregamento, pode-se recorrer a mapas base, poços próximos que possam ser utilizados para avaliar o posicionamento das linhas. Outra possibilidade é através do cruzamento entre linhas que poderá ser usado para avaliar se as coordenadas utilizadas são confiáveis. Um possível mapa guia pode ser o WebMaps de acesso livre no site do BDEP. Vale ressaltar que com o um montante de aproximadamente 30TB de dados sísmicos post-stack carregados ao longo dos anos no PetroBank, algumas inconsistências podem existir. $\mathrm{O}$ Webmaps utiliza a base de dados do PetroBank. 
Essas inconsistências são mais comuns em linhas antigas devido à, dentre uma das razões, a padronização da SEG ter sido alterada ao longo dos anos. No projeto em que este trabalho se insere, o carregamento foi realizado com o default do programa, e por isso, acabou utilizando as coordenadas da fonte e após a verificação com o mapa base, coincidiu de estar carregado igual, o que nos fez assumir que estava correto.

Após o carregamento, o trabalho de interpretação tornouse difícil por razões óbvias, já que a geologia não estava coerente entre as seções sísmicas. Foi necessária uma reavaliação em diversas etapas anteriores até chegar à inconsistência, demandando tempo para refazer boa parte das etapas posteriores como correção de mistie, correlação entre falhas, continuidade de refletores entre as seções sísmicas e etc.

\section{Agradecimentos}

Os autores agradecem à GEOPARK pelo financiamento do projeto RECAMU, à FAPESB e CAPES por bolsas de Pós-Graduação em Geofísica da UFBA. M.H. agradece ao $\mathrm{CNPq}$ por bolsa de produtividade em pesquisa ( $\mathrm{PQ}$ 304657/15-8).

\section{Referências}

Barry, K. M., Cavers, D. A. and Kneale, C. W., 1975, Report on recommended standards for digital tape formats: Geophysics, 40, no. 02, 344-352.

Brown, A. (2001). Calibrate yourself to your data! A vital first step in interpretation. Geophysical Prospecting 49, $p$ 729-733.

Cui, T. \& Margrave G. F. 2015. Drift time estimation by dynamic time warping. GeoConvention 2015 Geoscience new horizons.

Faust, L. Y. 1953. A velocity function including lithological variation. Geophysics 18, 271-88.

Gardner, G.H.F.; Gardner L.W.; Gregory A.R. (1974). "Formation velocity and density -- the diagnostic basics for stratigraphic traps" (PDF). Geophysics 39: 770780.

Norris, M.W.; Faichney, A.K. (Eds.) (2002). SEG Y rev1 Data Exchange format. Tulsa, OK: Society of Exploration Geophysicists. 12-20.

Theys, P, 1999. Log Data Acquisition and Quality Control. Second edition: Editions Technip. 3-4.

\begin{tabular}{|c|c|c|c|c|c|c|c|c|c|c|c|}
\hline \multicolumn{5}{|c|}{ Informação geral } & \multicolumn{2}{|c|}{ Validação do dado } & \multicolumn{5}{|c|}{ Controle de qualidade } \\
\hline $\begin{array}{c}\text { Nome do } \\
\text { levantamento }\end{array}$ & Tipo & Segmento & Ano & Datum & $\begin{array}{l}\text { Tempo } \\
\text { de } \\
\text { registro } \\
\text { (s) }\end{array}$ & $\begin{array}{c}\text { Taxa de } \\
\text { amostra- } \\
\text { gem } \\
\text { (ms) }\end{array}$ & $\begin{array}{c}\text { Múltiplas } \\
\text { * }\end{array}$ & $\begin{array}{c}\text { Difrações } \\
\text { * }\end{array}$ & $\begin{array}{c}\text { Sorrisos } \\
\text { de } \\
\text { migração } \\
*\end{array}$ & $\begin{array}{c}\text { Padrão de } \\
\text { refletores } \\
* *\end{array}$ & Observações \\
\hline $\begin{array}{c}\text { BAIA_TODOS } \\
\text { _SANTOS }\end{array}$ & MIG FIN & $0123-4567$ & & ARATU & 5,2 & 4 & Não & Não & Não & Bom & $\begin{array}{l}\text { Linha marinha de } \\
\text { boa qualidade. }\end{array}$ \\
\hline $\begin{array}{c}\text { BAIA_TODOS } \\
\text { _SANTOS }\end{array}$ & STK FIN & $0123-4568$ & & ARATU & 5,2 & 4 & Sim & Não & Não & Médio & $\begin{array}{l}\text { Possui ruído linear } \\
\text { mediante a } \\
\text { filtragem. }\end{array}$ \\
\hline $\begin{array}{c}\text { JEQUITINHONHA } \\
\text { _JACUIPE }\end{array}$ & MIG FIN & $0456-7890$ & 2003 & $\begin{array}{l}\text { SAD- } \\
69\end{array}$ & 4,1 & 4 & Sim & Sim & Sim & Médio & $\begin{array}{l}\text { Aconselhável } \\
\text { reprocessamento } \\
\text { (migração mal } \\
\text { sucedida) }\end{array}$ \\
\hline $\begin{array}{c}\text { JEQUITINHONHA } \\
\text { _JACUIPE }\end{array}$ & MIG FIN & $0456-7891$ & 2003 & $\begin{array}{l}\text { SAD- } \\
69\end{array}$ & 4,1 & 4 & Não & Não & Sim & Ruim & $\begin{array}{c}\text { Aconselhável } \\
\text { ganho nas } \\
\text { amplitudes e } \\
\text { reprocessamento. }\end{array}$ \\
\hline BAHIA_SUL & MIG FIN & $2345-6789$ & 2001 & $\begin{array}{c}\text { SAD- } \\
69\end{array}$ & 5 & 4 & Não & Não & Não & Bom & $\begin{array}{l}\text { Linha marinha de } \\
\text { boa qualidade. }\end{array}$ \\
\hline
\end{tabular}

Tabela 1: Exemplo de tabela de controle de qualidade dos dados sísmicos com informações gerais e que permitem a validação dos dados. 


\begin{tabular}{|c|c|c|c|c|c|c|c|}
\hline \multirow{2}{*}{$\begin{array}{c}\text { Nome do } \\
\text { poço }\end{array}$} & \multirow{2}{*}{ Setor } & \multicolumn{2}{|c|}{ Prof. Final } & \multirow{2}{*}{$\begin{array}{c}\text { Perfis } \\
\text { disponíveis }\end{array}$} & \multirow{2}{*}{ Observações } & \multicolumn{2}{|c|}{ Coord. UTM SAD69 24S } \\
\hline & & (m) & Unidade & & & $x$ & $\mathbf{Y}$ \\
\hline Poço 1 & Sul & 2191,9 & Fm. Sergi & SP, CÁLIPER & & 123456,7 & 9876543 \\
\hline Poço 2 & Leste & 2827 & $\begin{array}{c}\text { Fm. } \\
\text { Aliança }\end{array}$ & $\begin{array}{c}\text { GR, SP, } \\
\text { CÁLIPER, DT, } \\
\text { RHOB }\end{array}$ & $\begin{array}{c}\text { Testemunho } 890 \\
\text { m, } 1450 \mathrm{~m}\end{array}$ & 666666 & 7777777 \\
\hline Poço 3 & Leste & 2343 & Mb. Gomo & $\begin{array}{l}\text { GR, CÁLIPER, } \\
\text { DT, RILD }\end{array}$ & & $\cdots$ & $\cdots$ \\
\hline Poço 4 & Sul & 970 & Fm. Sergi & SP, CÁLIPER & $\begin{array}{c}\text { Desmorona- } \\
\text { mento } 420-450 \mathrm{~m}\end{array}$ & $\cdots$ & $\ldots$ \\
\hline Poço 5 & Sul & 2937 & & GR, CÁLIPER & & $\ldots$ & $\ldots$ \\
\hline
\end{tabular}

Tabela 2: Exemplo de tabela para controle de qualidade de poços com informações relevantes para cada poço. 